Math. Z.121, $190(1971)$

(C) by Springer-Verlag 1971

\title{
Correction to \\ "Collineations of Finite Projective Spaces as Permutations on the Sets of Dual Subspaces"
}

Math. Z. 111, 249-254 (1969)

ASCHER WAGNER

I am indebted to $H$. Lüneburg for pointing out to me that expression (15) of the above paper is nonsense. This invalidates the next one and a half pages of that paper which are devoted to showing - which is crucial to the proof that $\operatorname{det}\left(\mathbf{M}_{t}\right) \neq 0$.

In fact $\operatorname{det}\left(\mathbf{M}_{t}\right) \neq 0$ as we shall now show by proving that $\operatorname{det}\left(\mathbf{M}_{t}\right)=q^{(t+1) r}$. We first note that

$$
q^{i(v-u+1)} b_{u, v+1}-q^{i} \cdot q^{i(v-u)} b_{u, v}=q^{(i+1)(v-u+1)} b_{u-1, v}
$$

as may easily be verified by direct computation.

We now reduce $\operatorname{det}\left(\mathbf{M}_{t}\right)$ to triangular form as follows:

$1^{\text {th }}$ step. Subtract the $k^{\text {th }}$ column from the $(k+1)^{\text {th }}$ column where $k$ takes the value $t+1, t, \ldots, 1$ in turn. (This replaces the first row by $1,0, \ldots, 0$, also it leaves the 1's on the main diagonal unchanged and replaces a typical term $b_{u, v}$ by $q^{v-u} b_{u-1, v-1}$ )

$i^{\text {th }}$ step. Subtract $q^{i-1}$ times the $k^{\text {th }}$ column from the $(k+1)^{\text {th }}$ column where $k$ takes the values $t+1, t, \ldots, i$ in turn. (This replaces the $i^{\text {th }}$ row by $0, \ldots, 0,1,0, \ldots, 0$ and replaces a typical term $q^{(i-1)(v-u)} b_{u-i+1, v-i+1}$ by $\left.q^{i(v-u)} b_{u-i, v-i}\right)$

After $t+1$ steps we obtain a triangular determinant with zeros above the main diagonal and diagonal entries $1,1, \ldots, 1, q^{(t+1) r} b_{-1, r-1}$.

Hence $\operatorname{det}\left(\mathbf{M}_{t}\right)=q^{(t+1) r} b_{-1, r-1}=q^{(t+1) r}$.

\author{
Dr. Ascher Wagner \\ Queen Elizabeth College \\ Campden Hill Road \\ London $\mathrm{W} 8$ \\ England
}

